\title{
VARIACIÓN ESTACIONAL DE LA COMPOSICIÓN QUÍMICA DEL ACEITE ESENCIAL DE LAS HOJAS DE Ophryosporus peruvianus
}

\author{
Edith María Fernanda Mattos Vila, Ingrit Elida Collantes Díaz*
}

\begin{abstract}
RESUMEN
El arbusto efímero Ophryosporus peruvianus fue recolectado por cinco meses (diciembre 2018 hasta abril 2019) en Andahuaylillas (Cusco). El aceite esencial de las hojas del arbusto fue obtenido por hidrodestilación y analizado por cromatografía gaseosa acoplado a espectrómetro de masas. Fueron identificados 89 componentes del aceite esencial entre monoterpenos oxigenados, hidrocarburos sesquiterpénicos, sesquiterpenos oxigenados, diterpenos oxigenados, entre otros, de los cuales los componentes mayoritarios fueron los hidrocarburos sesquiterpénicos $\beta$-cariofileno, (Z)- $\beta$-farneseno y $\beta$-sesquifelandreno y estuvieron presentes en las muestras de los 5 meses de colecta.
\end{abstract}

Palabras clave: aceite esencial, variación estacional, sesquiterpenos

\section{SEASONAL VARIATION OF THE CHEMICAL CONSTITUENTS OF THE ESSENTIAL OIL OF THE LEAVES FROM Ophryosporus peruvianus}

\begin{abstract}
The ephemeral shrub Ophryosporus peruvianus was collected for five months (December 2018 - April 2019) in Andahuaylallas (Cusco). The oil essential from leaves of the bush was obtained by hydrodistillation and analyzed by gas chromatography coupled to a mass spectrometer. Eigthy nine components of the essential oil were identified among oxygenated monoterpenes, sesquiterpenes hydrocarbons, sesquiternes oxygenated, oxygenated diterpenes, among others, of which the major components were the sesquiterpenes hydrocarbons $\beta$-caryophyllene, (Z)- $\beta$-Farnesene y $\beta$-Sesquiphellandrene and were presents in the samples of the five months of collection.
\end{abstract}

Key words: essential oil, seasonal variation, sesquiterpenes

Facultad de Ingeniería Química y Textil, Universidad Nacional de Ingeniería. Av. Túpac Amaru º 210, Rímac, Lima-Perú, ingrit_uni@hotmail.com 


\section{INTRODUCCIÓN}

Los aceites esenciales son mezclas complejas de compuestos lipofílicos, que se caracterizan por ser de baja masa molar y volátiles, en general estos compuestos juegan un rol ecológico importante en las plantas como protección contra microorganismos y herbívoros, atrayentes de polinizadores y de comportamientos aleloquímicos ${ }^{1}$. La biosíntesis, la composición y la producción de los aceites esenciales pueden ser afectadas por factores bióticos y abióticos ambientales. En el contexto de la composición química y producción del aceite esencial, pueden ser diferentes entre los órganos de la misma planta y entre las diferentes fases estacionales (primavera, otoño, invierno y verano) del desarrollo de la planta ${ }^{2,3}$. Más de 1000 compuestos orgánicos volátiles ya fueron identificados y consisten de aldehídos, alcoholes, ésteres y varios tipos terpenos ${ }^{2}$.

Ophryosporus peruvianus es un arbusto que puede ser encontrado entre los meses de diciembre a abril, es por esa razón que es denominada una planta efímera ${ }^{4}$, esta especie pertenece al género Ophryosporus de la familia Asteraceae, familia que cuenta con aproximadamente 950 géneros y 20000 especies $^{5}$. El género Ophryosporus presenta alrededor de 48 especies en el mundo y en el Perú se han registrado 21 especies 6 .

La especie $O$. peruvianus puede ser localizada en las regiones de la sierra del Perú, dependiendo de su localización recibe varios nombres populares y es usada en medicina popular para diferentes males dependiendo de su origen local ${ }^{7,8}$. Del aceite esencial de las partes aéreas fue aislado e identificado epoxicariofileno del aceite esencial ${ }^{7}$ y de las partes aéreas fueron aislados e identificados 3-[3,3-dimetilalil]-5-senecioil-p-hidroxi-acetofenona; 6-acetil-8-isovaleril-2,2-dimetil-cromano y 3-isovateril-5-[1-hidroxi-3,3-dimetilalil]-phidroxiacetofenona ${ }^{9}$

El presente trabajo es un primer reporte de la variación estacional de la composición química de los aceites esenciales de las hojas de Ophryosporus peruvianus colectados en el distrito de Andahuaylillas (Cusco) entre los meses de diciembre del 2018 hasta abril de 2019.

\section{PARTE EXPERIMENTAL}

\section{Colecta del material vegetal}

Las partes aéreas de $O$. peruvianus fueron colectadas en el distrito de Andahuaylillas, provincia de Quispicanchi departamento del Cusco a una altura de $3152 \mathrm{msnm}$. La colecta fue realizada mes a mes desde diciembre del 2018 hasta abril del 2019. El material vegetal para la identificación botánica fue colectado en el mes de febrero del 2019, mes en que la planta tenía las flores presentes. La identificación botánica fue realizada por el Museo de Historia Natural de la Universidad Nacional Mayor de San Marcos según el Sistema de Clasificación de Croquist (1988) y fue clasificada que pertenece a la división Magnoliophyta, clase Magnoliopsida, subclase Asteridae, orden Asterales, familia Asteraceae, género Ophryosporus, especie Ophryosporus peruvianus (J.F.Gmel) R. King \& H. Rob. La especie 
es conocida con los nombres populares arenilla, puschca, chichipia, té inka, cushpishkai, chichi casha, chichis, japia-japia, kulpishka y puede ser localizada en Canta, Huarochirí, Yauyos, Cusco, región Ancash en el distrito de Pamparomás y Cajatambo en las localidades de Cruzjirca, La Florida, Anthay y Astobamba.

La masa de hojas colectadas en diciembre del 2018 fue de 215,22 g, en enero, febrero, marzo y abril del 2019 fue de 330 g, 260 g, 991 g y 974 g, respectivamente.

\section{Extracción de los aceites esenciales}

Las hojas fueron separadas de los tallos y los aceites esenciales fueron obtenidos por hidrodestilación de 4 horas con aparato tipo Clevenger (Farmacopea), el aceite esencial fue extraído con éter de petróleo (EP) y secado con sulfato de sodio anhidro, el EP fue evaporado a temperatura de ambiente y el aceite fue pesado para determinar su rendimiento y guardado en congeladora $\left(-20^{\circ} \mathrm{C}\right)$ hasta su análisis por cromatografía gaseosa acoplada a espectrometría de masas $(\mathrm{CG}-\mathrm{EM})^{2}$.

\section{Análisis de los aceites esenciales}

Las muestras del aceite esencial fueron preparadas a una concentración de $1 \mathrm{mg} / \mathrm{mL}$. El análisis por cromatografía gaseosa fue realizado en un cromatógrafo a gas acoplado a un espectrómetro de masas (Shimadzu, modelo CGMS-QP2010 Ultra) con las siguientes condiciones de análisis: columna capilar Restek Rtx-5MS (30 m x 0,25 mm x 0,25 $\mu \mathrm{m}$ ); temperatura del inyector $260{ }^{\circ} \mathrm{C}$, temperatura del detector $280{ }^{\circ} \mathrm{C}$; impacto de electrones a $70 \mathrm{eV}$, con gas de arrastre helio a un flujo de $1,48 \mathrm{~mL} / \mathrm{min}$, con split 1/5; con un programa de temperatura de $80^{\circ} \mathrm{C}\left(5^{\prime}\right)-280^{\circ} \mathrm{C}\left(5^{\prime}\right), 8^{\circ} \mathrm{C} /$ minuto; y con volumen de inyección de $3 \mu \mathrm{L}$. La identificación de la composición química fue basada por la comparación de los espectros de masas con el banco de datos del sistema de CG-EM de NIST 14 lib y por análisis de sus espectros $^{10}$.

\section{RESULTADOS Y DISCUSIÓN}

La hidrodestilación de las hojas de $O$. peruvianus produjo aceites esenciales de color amarillo transparente con presencia de cristales amarillos, los rendimientos fluctuaron como se puede apreciar en la tabla 1. De los meses de marzo y abril se obtuvo menores rendimientos debido a que la planta estaba más húmeda con respecto a los otros meses.

El análisis por cromatografía gaseosa acoplada a espectrómetro de masas de los aceites esenciales permitió la identificación de 89 compuestos. Los componentes mayoritarios son $\beta$-Cariofileno, (Z)- $\beta$-Farneseno, (E)- $\beta$-Farnesene, aromadendrano, $\gamma$-Curcumeno, Germacreno $D$, biciclogermacreno, $\beta$-Sesquifelandreno, tran-Sesquisabineno hidrato, óxido de Cariofileno, $\beta$-Bisabolol, $11 \mathrm{H} \alpha$-Himachal-4-en-1 $\beta$-ol, $\alpha$-Vetivono, encecalina y 2',3',3'-trimetil-2',3'-Dihidroangelicina, de los cuales se destacan $\beta$-cariofileno, (Z)- $\beta$ farneseno y $\beta$-sesquifelandreno (tabla 1). Analizando la composición química se puede observar la presencia de monoterpenos oxigenados (1-8, 11-13, 16 y 18 ), ésteres alifáticos 
(9,10 y 14), alilbencenos $(17,23)$, hidrocarburos sesquiterpénicos $(15,19-22,24-32,34-42$ y 45-46), cromenos $(33,61,76$ y 77), sesquiterpenos oxigenados (43-44, 47-60, 62-67, 69, 70, 72-75, 78 y 80), aldehído alifáticos (68), los diterpenos oxigenados (79, 81-83 y 8586) y los alkanos $(84,87-89)$ (figura 1). Al analizar el total de los componentes químicos identificados (tabla 1) se observa que los hidrocarburos sesquiterpénicos son los que están presentes en mayor proporción, seguida de los sesquiterpenos oxigenados y cromenos, los demás compuestos están en baja proporción.

El último mes de colecta (abril 2019) presenta 11 compuestos que no están presentes en los meses anteriores (tabla 1) que son los monoterpenos oxigenados (1-5), hexil isovalerato (10), sesquicineole (43), acetato de occidol (80), acetato de cis-ferruginol (85), 6-dioxi-Taxodione (86) y pentacosano (87), todos ellos en bajo porcentaje. Este hecho coincide con el último mes en que es posible encontrar $O$. peruvianus ya que es una planta efímera ${ }^{4}$.

Tabla 5. Porcentajes de rendimiento de los aceites esenciales, así como, los componentes identificados, tiempo de retención y el porcentaje en el aceite esencial de las hojas de O. peruvianus.

\begin{tabular}{|c|c|c|c|c|c|c|c|}
\hline \multirow{2}{*}{\multicolumn{2}{|c|}{ Porcentaje de rendimiento (\%) }} & & \multirow{2}{*}{$\begin{array}{l}\text { Dic } \\
0,53\end{array}$} & \multirow{2}{*}{$\begin{array}{c}\text { Ene } \\
0,4 \\
\end{array}$} & \multirow{2}{*}{\begin{tabular}{|l|} 
Feb \\
0,43 \\
\end{tabular}} & \multirow{2}{*}{$\begin{array}{l}\text { Mar } \\
0,15 \\
\end{array}$} & \multirow{2}{*}{$\begin{array}{l}\text { Abr } \\
0,28\end{array}$} \\
\hline & & & & & & & \\
\hline & Nombre & TR & & & & & \\
\hline 1 & Alcohol de yomogi & 4,428 & 0 & 0 & 0 & 0 & 0,02 \\
\hline 2 & Linalool & 7,114 & 0 & 0 & 0 & 0 & 0,05 \\
\hline 3 & trans-Sabineno hidrato & 7,490 & 0 & 0 & 0 & 0 & 0,08 \\
\hline 4 & cis- $p$-Ment-2-en-1-ol & 7,685 & 0 & 0 & 0 & 0 & 0,05 \\
\hline 5 & trans- $p$-Menta-2,8-dien-1-ol & 7,935 & 0 & 0 & 0 & 0 & 0,09 \\
\hline 6 & cis- $\alpha-N e c r o d o l$ & 8,610 & 0 & 0,06 & 0 & 0 & 0,07 \\
\hline 7 & Terpinen-4-ol & 9,039 & 0,09 & 0,34 & 0,06 & 0,14 & 0,33 \\
\hline 8 & $\alpha$-Terpineol & 9,338 & 0,03 & 0,08 & 0 & 0,04 & 0,07 \\
\hline 9 & (3Z)-Hexenil 3-metil butanoato & 10,309 & 0,05 & 0,13 & 0,07 & 0,03 & 0,13 \\
\hline 10 & Hexil isovalerato & 10,424 & 0 & 0 & 0 & 0 & 0,02 \\
\hline 11 & Acetato de cis-Crisantenil & 10,569 & 0 & 0,1 & 0 & 0,05 & 0,09 \\
\hline 12 & Acetato de Bornilo & 11,369 & 0 & 0 & 0 & 0,03 & 0,09 \\
\hline 13 & Acetato de Lavandulil & 11,428 & 0,04 & 0,25 & 0 & 0,1 & 0,14 \\
\hline 14 & (3Z)-Hexenil tiglato & 12,087 & 0,07 & 0,08 & 0,11 & 0,04 & 0,05 \\
\hline 15 & $\delta$-Elemeno & 12,371 & 0,04 & 0 & 0 & 0,04 & 0,07 \\
\hline 16 & Acetato de $\alpha$-Terpinil & 12,624 & 0 & 0,04 & 0 & 0 & 0,02 \\
\hline 17 & Eugenol & 12,716 & 0,19 & 0,06 & 0,32 & 0,15 & 0,58 \\
\hline 18 & Acetato de Neril & 12,815 & 0,27 & 0,53 & 0,18 & 0,49 & 0,29 \\
\hline 19 & $\alpha$-Ylangeno & 13,112 & 0,15 & 0,23 & 0,13 & 0,09 & 0,17 \\
\hline 20 & $\beta$-Bourboneno & 13,267 & 0,03 & 0,19 & 0,13 & 0,04 & 0,09 \\
\hline 21 & $\alpha$-Duprezianeno & 13,320 & 0,14 & 0,18 & 0 & 0,11 & 0,17 \\
\hline 22 & $\beta$-Elemeno & 13,375 & 0,26 & 0,31 & 0,1 & 0,22 & 0,12 \\
\hline 23 & Metil eugenol & 13,540 & 0,22 & 0,33 & 0,26 & 0,23 & 0,44 \\
\hline 24 & Italiceno & 13,606 & 0,38 & 0,43 & 0,28 & 0,2 & 0,28 \\
\hline 25 & Sesquitujeno & 13,772 & 0,04 & 0,04 & 0 & 0,03 & 0,06 \\
\hline 26 & $\beta$-Cariofileno & 13,900 & 14,85 & 12,45 & 14,33 & 15,22 & 15,53 \\
\hline 27 & $\beta$-Ylangeno & 14,030 & 0 & 0,04 & 0 & 0 & 0 \\
\hline 28 & trans- $\alpha$-Bergamoteno & 14,119 & 0,17 & 0,19 & 0,14 & 0,16 & 0,22 \\
\hline 29 & $(Z)$ - $\beta$-Farneseno & 14,252 & 14,94 & 16,99 & 17,36 & 13,61 & 14,22 \\
\hline 30 & $\alpha$-Himacaleno & 14,322 & 0,05 & 0,1 & 0 & 0,1 & 0,11 \\
\hline
\end{tabular}




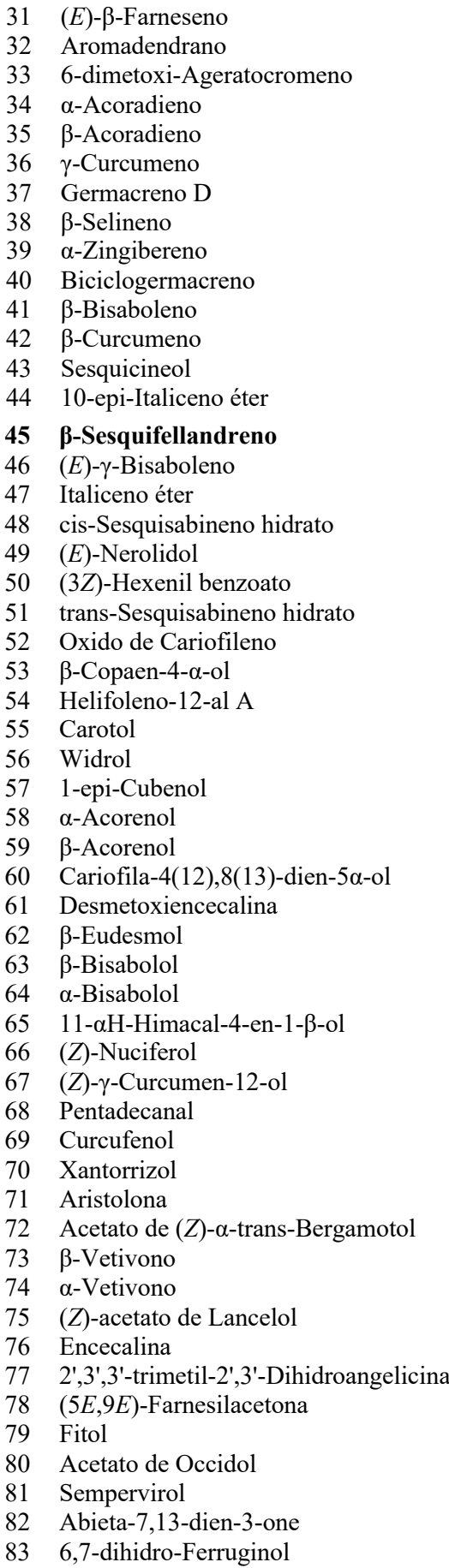

$\begin{array}{rrrrrr}14,430 & 1,32 & 1,28 & 0,91 & 1,12 & 0,94 \\ 14,473 & 0,96 & 1,3 & 1,03 & 1,05 & 1,07 \\ 14,561 & 0 & 0 & 0 & 0,03 & 0,08 \\ 14,635 & 0,08 & 0,08 & 0 & 0,04 & 0,07 \\ 14,680 & 0,08 & 0,11 & 0,05 & 0,07 & 0,09 \\ 14,845 & 5,32 & 1,49 & 4,72 & 4,23 & 4,04 \\ 14,922 & 4,87 & 0 & 4,74 & 6,25 & 4,33 \\ 15,017 & 0,18 & 0,15 & 0,11 & 0,16 & 0,2 \\ 15,093 & 0,41 & 0,16 & 0,2 & 0,35 & 0,4 \\ 15,175 & 1,23 & 0,39 & 1,02 & 1,35 & 0,78 \\ 15,310 & 0,35 & 0,35 & 0,24 & 0,3 & 0,33 \\ 15,359 & 0,33 & 0,16 & 0,29 & 0,29 & 0,41 \\ 15,410 & 0 & 0 & 0 & 0 & 0,04 \\ 15,468 & 0,16 & 0,23 & 0,23 & 0,21 & 0,26 \\ \mathbf{1 5 , 5 7 1} & \mathbf{1 6 , 2 5} & \mathbf{1 4 , 3 9} & \mathbf{9 , 7 3} & \mathbf{1 4 , 5 6} & \mathbf{1 3 , 2 5} \\ 15,690 & 0,03 & 0 & 0 & 0,06 & 0,09 \\ 15,771 & 0,35 & 0,46 & 0,53 & 0,45 & 0,45 \\ 15,865 & 0,63 & 0,76 & 0,79 & 0,63 & 0,69 \\ 16,140 & 0,45 & 0,66 & 0,37 & 0,65 & 0,81 \\ 16,275 & 0,06 & 0,16 & 0 & 0,07 & 0,12 \\ 16,421 & 1,68 & 2,85 & 2,76 & 2,43 & 2,23 \\ 16,549 & 1,39 & 3,22 & 2,03 & 1,72 & 1,92 \\ 16,718 & 0,2 & 0,3 & 0,34 & 0,28 & 0,39 \\ 16,840 & 0,15 & 0,21 & 0,18 & 0,32 & 0,3 \\ 16,927 & 0,58 & 1,19 & 0,73 & 0,72 & 0,97 \\ 17,052 & 0,06 & 0 & 0,07 & 0,08 & 0,22 \\ 17,176 & 0,46 & 0,52 & 0,34 & 0,54 & 0,65 \\ 17,237 & 0,42 & 0,89 & 0,46 & 0,37 & 0,53 \\ 17,282 & 0,26 & 0,34 & 0,27 & 0,26 & 0,37 \\ 17,329 & 0,28 & 0,54 & 0,46 & 0,35 & 0,46 \\ 17,491 & 0,11 & 0 & 0,14 & 0 & 0,21 \\ 17,532 & 0,55 & 0,77 & 0,4 & 1,17 & 0,62 \\ 17,777 & 1 & 1,08 & 1,02 & 1,08 & 1,25 \\ 17,946 & 0,51 & 0,57 & 0,51 & 0,58 & 0,06 \\ 18,005 & 1,93 & 2,95 & 1,88 & 2,62 & 2,27 \\ 18,131 & 0,23 & 0,39 & 0,27 & 0,3 & 0,37 \\ 18,239 & 0,22 & 0,44 & 0,21 & 0,2 & 0,31 \\ 18,303 & 0,25 & 0,21 & 0,33 & 0,09 & 0,27 \\ 18,376 & 0,03 & 0,06 & 0,08 & 0,05 & 0,1 \\ 18,848 & 0,17 & 0,14 & 0,07 & 0,19 & 0,11 \\ 19,109 & 0 & 0,1 & 0 & 0 & 0,11 \\ 19,418 & 0,04 & 0 & 0 & 0,03 & 0,07 \\ 19,555 & 0,05 & 0,09 & 0 & 0,03 & 0,12 \\ 20,325 & 3,67 & 1,54 & 5,24 & 2,78 & 2,13 \\ 20,608 & 0,07 & 0,07 & 0 & 0,05 & 0,11 \\ 21,473 & 5,5 & 5,07 & 9,15 & 6,75 & 5,81 \\ 21,956 & 1,24 & 1,58 & 1,71 & 2,07 & 1,88 \\ 22,360 & 0,03 & 0 & 0 & 0 & 0,02 \\ 23,327 & 0,37 & 0,51 & 0,25 & 0,31 & 0,4 \\ 23,772 & 0 & 0 & 0 & 0 & 0,03 \\ 23,992 & 0,08 & 0,09 & 0,13 & 0,1 & 0,09 \\ 24,530 & 0,07 & 0,05 & 0,1 & 0,11 & 0,08 \\ 24,991 & 0,08 & 0,07 & 0,07 & 0,07 & 0,06 \\ & & & & & \end{array}$




\begin{tabular}{|c|c|c|c|c|c|c|c|}
\hline 84 & Tetracosano & 25,324 & 0,03 & 0 & 0 & 0 & 0,03 \\
\hline 85 & Acetato de cis-Ferruginol & 25,540 & 0 & 0 & 0 & 0 & 0,04 \\
\hline 86 & 6-dioxi-Taxodion & 25,749 & 0 & 0 & 0 & 0 & 0,03 \\
\hline 87 & Pentacosano & 27,348 & 0 & 0 & 0 & 0 & 0,02 \\
\hline 88 & Hexacosano & 29,230 & 0,11 & 0,14 & 0,14 & 0,1 & 0,12 \\
\hline 89 & Heptacosano & 31,093 & 0,1 & 0,2 & 0,18 & 0,13 & 0,36 \\
\hline & Monoterpenos oxigenados (\%) & & 0,43 & 1,4 & 0,24 & 0,85 & 1,39 \\
\hline & Ésteres alifáticos $(\%)$ & & 0,12 & 0,21 & 0,18 & 0,07 & 0,2 \\
\hline & Alilbencenos (\%) & & 0,41 & 0,39 & 0,58 & 0,38 & 1,02 \\
\hline & Hidrocarburos sesquiterpénicos (\%) & & 62,46 & 51,01 & 55,51 & 59,65 & 57,04 \\
\hline & Cromenos $(\%)$ & & 6,85 & 6,65 & 11 & 8,85 & 7,98 \\
\hline & Sesquiterpenos oxigenados (\%) & & 15,63 & 20,43 & 19,24 & 18,16 & 17,98 \\
\hline & Aldehídos alifáticos (\%) & & 0,25 & 0,21 & 0,33 & 0,09 & 0,27 \\
\hline & Diterpenos oxigenados (\%) & & 0,60 & 0,72 & 0,55 & 0,59 & 0,70 \\
\hline & Alkanos (\%) & & 0,24 & 0,34 & 0,32 & 0,23 & 0,53 \\
\hline & Total de compuestos identificados (\%) & & 86,99 & 81,46 & 87,95 & 88,87 & 87,22 \\
\hline
\end{tabular}

TR: tiempo de retención, Dic: diciembre, Ene: enero, Feb: febrero, Mar: marzo, Abr: abril.

En los cinco meses de colectas realizadas, fue observado 15 compuestos mayoritarios y dentro de este grupo (figura 1), fue constante la presencia de tres sesquiterpenos $\beta$-cariofileno $(12,45-15,53 \%)$, (Z)- $\beta$-farneseno (13,61-17,36 \%) y $\beta$-sesquifelandreno $(9,73-16,25 \%)$, el primer mes de la colecta fue observado que $\beta$-sesquifelandreno es el mayoritario $(16,25 \%)$, el segundo y tercer mes de colecta se observó (Z)- $\beta$-farneseno como el mayoritario y son los más altos porcentajes observados en la composición del aceite y el penúltimo y último mes de colecta fue el $\beta$-cariofileno el de mayor porcentaje. Los tres sesquiterpenos representa 46,04 $\%$ para el mes de diciembre, 43,83 \% enero, 41,42\% febrero, 43,39\% marzo y $43 \%$ abril.

De los doce restantes componentes mayoritarios se observó que la encecalina está presente en los 5 meses y en febrero está en mayor porcentaje $(9,15 \%)$, lo que no se puede decir del germacreno $\mathrm{D}$, no está presente en el mes de enero. $\gamma$-curcumeno y $\alpha$-vetivono, están presentes todos los meses de colecta, observando que en el mes de enero presentan los más bajos porcentajes ( $1,49 \%$ y $1,54 \%$, respectivamente).

Para el trans-sesquisabineno hidrato y el óxido de cariofileno sus máximos valores de porcentaje de presencia fueron alcanzados en los meses de enero y febrero. Los máximos valores de 11- $\alpha$ H-Himacal-4-en-1- $\beta$-ol son alcanzados en el mes de enero y marzo, finalmente 2',3',3'-trimetil-2',3'-Dihidroangelicina alcanza sus máximos valores en los meses de febrero y marzo.

Montenegro (2001) trabajó con el aceite esencial de las partes aéreas de O. peruvianus colectados en Yauyos, en este trabajo fue aislado el óxido de cariofileno, dato con el que presume que fue el compuesto mayoritario; comparando el estudio del 2001 con el presente trabajo, se observa que el óxido de cariofileno no es uno de los tres mayoritarios sesquiterpenos. De acuerdo a la literatura ${ }^{2,3}$ la composición de los aceites esenciales se puede ver afectado por factores bióticos y abióticos del medio ambiente, la planta fue colectada 
en diferentes lugares. La composición química puede variar de acuerdo a los órganos de la planta $^{2,3}$, Montenegro uso partes aéreas (hojas, ramas y flores), mientras que en el presente estudio se usaron sólo hojas.

En los estudios de aceite esencial de las partes aéreas de otras especies del mismo género, O. heptanthus ${ }^{11}$, O. pinifolius $^{12}$ y en $O$. piquerioides $^{13}$ presentaron monoterpenos no oxigenados y oxigenados como mayoritarios, lo que no sucede en la especie objetivo de nuestro estudio. De los tres sesquiterpenos mayoritarios presentes en $O$. peruvianus, sólo $\beta$-cariofileno también está presente en $O$. pinifolius y $O$. piquerioides, con la diferencia que no corresponde a los mayoritarios en esas especies.

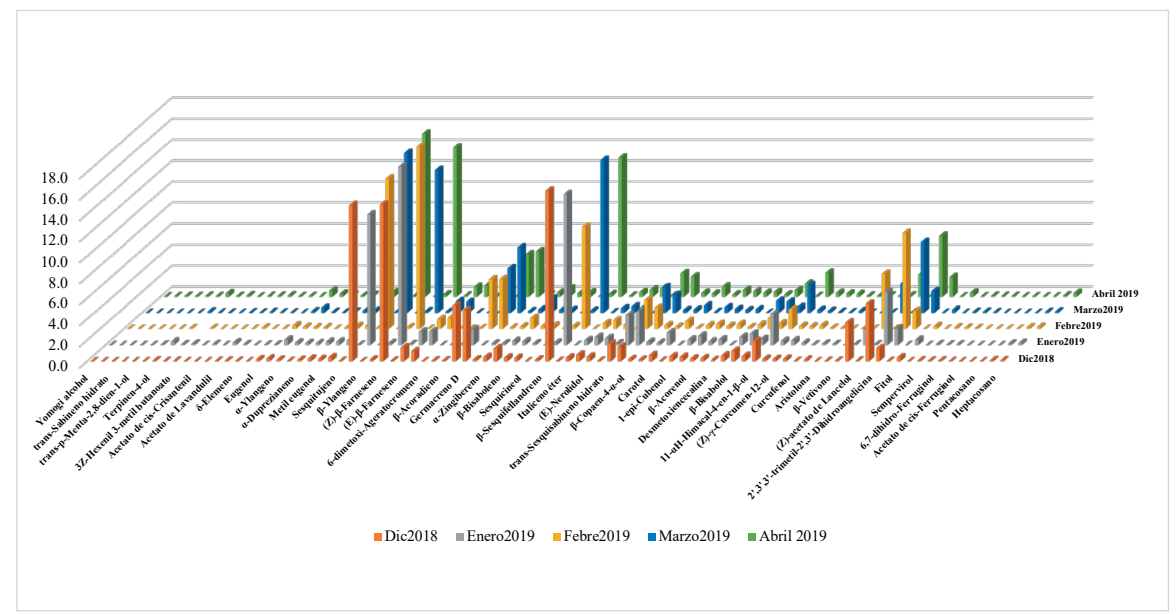

Figura 1. Variación estacional de la composición química del aceite esencial de las hojas de $O$. peruvianus.

El sesquiterpeno bicíclico natural $\beta$-cariofileno es comúnmente encontrado en los aceites esenciales de plantas como Cinnamomum tamala (25,3,\%), Didymocarpus tomentosa $(78,7, \%)$, tiene actividad antioxidante, antibacteriano, anticancerígeno, antiinflamatorio, cardioprotector, hepatoprotector, gastroprotective, nefroprotectivo ${ }^{14}$.

(Z)- $\beta$-farneseno es un hidrocarburo sesquiterpénico acíclico con actividad insecticida y es atóxico para plantas, animales superiores y el hombre. También fue reportado su actividad antioxidante ${ }^{15}$.

$\beta$-sesquifelandreno es un sesquiterpeno que presenta actividad anticancerígena ${ }^{16}$.

La biosíntesis de los sesquiterpenos $\beta$-cariofileno, $(Z)$ - $\beta$-farneseno y $\beta$-sesquifelandreno se llevan a cabo en el citosol a través de la vía del mevalonato ${ }^{14}$. 


\section{CONCLUSIONES}

El aceite esencial obtenido por el método de hidrodestilación de las hojas de Ophryosporus peruvianus colectados en Andahuaylillas durante la estación de diciembre-2018 a abril-2019 presenta una composición química muy variada desde monoterpenos oxigenados, hidrocarburos sesquiterpénicos, sesquiterpenos oxigenados, diterpenos oxigenados. Los metabolitos secundarios mayoritarios presentes en los cinco meses de colecta fueron los hidrocarburos sesquiterpénicos $\beta$-cariofileno, (Z)- $\beta$-farneseno y $\beta$-sesquifelandreno.

\section{AGRADECIMIENTO}

Los autores agradecen el financiamiento del Vicerrectorado de Investigación de la Universidad Nacional de Ingeniería, Proyecto de Investigación Formativa 2019 (FIQT-MF-2-2019).

\section{REFERENCIAS BIBLIOGRÁFICAS}

1. Fürstenberg-Hägg J, Zagrobelny M, Bak S. Plant defense against insect herbivores. Int J Mol Sci. 2013; 14: 10242 - 10297.

2. De oliveira TS, Bombo AB, De oliveira ASS, Garcia VL, Appezzato da GB. Seasonal variation of the essential oil two Brazilan native Aldama La LLave (Asteraceae) species. An Acad Bras Cienc. 2016; 88(3 suppl.): 1899-1907.

3. Deschamps C, Zanatta J, Bizzo H, Oliveira M, Roswalka L. Seasonal evaluation of essential oil yield of mint species. Ciênc Agrotec. 2008; 32 (3): 725 -730.

4. Guzmán-Lúcio MA, Wendt T, Simpson B, Alvarado-Vázquez MA, ForoughbakhchPournavab R, González-Álvarez M, Rocha-Estrada A. Listado florístico de especies anuales de floración invernal en el noreste de Nuevo León, México. Rev Mex Biodivers. 2013; 84: $884-893$.

5. Rahman AHMM, Alam MS, Khan SK, Ahmed F, Islam AKMR, Rahman MM. Taxonomic studies on the family Asteraceae (Compositae) of the Rajshahi Division. Res J Agric \& Biol Sci. 2008; 4(2): 134 - 140.

6. Sagástegui Alva A, Rodríguez Rodríguez EF. Una nueva especie de Ophryosporus (Eupatorieae: Asteraceae) para el Perú. Rev Perú Biol. 2008; 15(1): 21 - 23.

7. Montenegro LCA. Estudio Químico del Aceite Esencial del Te Inka (Ophryosporus peruvianus). [Tesis de licenciatura]. Lima: Universidad Nacional de Ingeniería; 2001.

8. Quinteros GYM. Etnobotánica y revaloración de los conocimientos tradicionales de la flora medicinal en Cajatambo, Lima. [Tesis de maestria]. Lima: Pontificia Universidad Católica del Perú. 2009.

9. Bohlmann F, Wallmeyer M, King RM, Robinson H. 2-Oxo-labda-8(17),13-Dien-15-ol from Ophryosporus chilca. Phytochem. 1984; 23(4): 1513 - 1514

10. Adams RP. Identification of essential oil components by gas chromatography/mass spectrometry. 4th Ed. Carol Stream, Illinois, USA: Allured Publishing Corporation. 2007. 
11. Arze JBL, Collin G, Garneau F-X, Jean F-I, Gagnon H. Essential oil from Bolivia II. Asteraceae: Ophryosporus heptanthus (Wedd.) H. Rob. Et King. J Essent Oil Res. 2004; 16(4): $374-376$.

12. Niemeyer H. Composition of essential oils from five aromatic species of Asteraceae. $\mathrm{J}$ Essent Oil Res. 2009; 21(4): 350 - 353.

13. Garneau F-X, Collin GJ, Jean F-I, Gagnon H, Arze JBL. Essential oil from Bolivia XII Asteraceae: Ophryosporus piquerioides (D.C.) Benth. ex Baker. J Essent Oil Res. 2013; 25(5): $388-394$.

14. Machado K Da C, Islam MT, Ali ES, Rouf R, Uddin SJ, Dev S, et al. A Systematic review on the neuroprotective perspectives of beta-caryophyllene. Phytother Res. 2018; 32(12): $2376-2388$.

15. Peña-Montes DJ, Huerta-Cervantes M, Ríos-Silva M, Trujillo X, Huerta M, et al. Protective effect of the hexanic extract of Eryngium carlinae Inflorescences In vitro, in Yeast, and in Streptozotocin-Induced diabetic male rats. Antioxidants (Basel). 2019 Mar 26;8(3):73. doi: 10.3390/antiox8030073.

16. Tyagi AK, Prasad S, Yuan W, Li S, Aggarwal BB. Identification of a novel compound ( $\beta$-sesquiphellandrene) from turmeric (Curcuma longa) with anticancer potential: comparison with curcumin. Invest New Drugs.. 2015; 33: 1175 - 1186. 